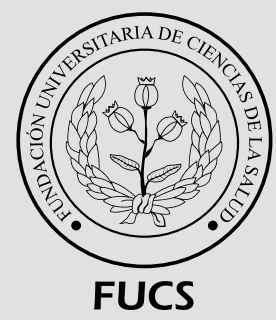

\title{
Índice de manejo quirúrgico en pacientes con cáncer ginecológico durante la pandemia por Covid-19: propuesta para instituciones y profesionales en ginecología oncológica
}

Alexander Rodríguez MD $^{\mathrm{a}}$ Luis Orlando Puentes MD Jairo Mendoza MD' Marcela Valencia Franco MD. MSc ${ }^{d}$

Index of surgical management in gynecological cancer patients during the covid-19 pandemic: a proposal for institutions and gynecological onchology professionals

${ }^{a}$ Ginecólogo Oncólogo, Centro Oncológico de Antioquia. Docente Universidad CES. Presidente Asociación Antioqueña de Ginecología y Obstetricia. Asagio. Medellín, Colombia.

${ }^{b}$ Ginecólogo Oncólogo, Fundación Universitaria de Ciencias de la Salud, Hospital de San José y Hospital San Ignacio, Bogotá DC, Colombia.

' Ginecólogo Oncólogo, Hospital Occidente de Kennedy, Bogotá, Colombia.

${ }^{d}$ MSc Salud Pública, Departamento de salud pública, Universidad CES, Medellín.

\section{R E S U M E N}

El mundo vive una pandemia por un Coronavirus, llamado SARS-COV-2, que produce la COVID-19 (acrónimo del ingles coronavirus disease 2019), la cual ha generado un colapso en los sistemas de salud, haciendo que el manejo de otras enfermedades se convierta en un reto. De igual forma para los grupos oncológicos, la presencia de esta enfermedad, genera muchas dudas en la aplicación de los tratamientos estándares, los cuales se deben realizar lo antes posible, con el fin de ofrecer mejores resultados oncológicos.

Se propone la creación de un índice ( COVID-19 Cáncer Index), teniendo en cuenta variables clínicas, epidemiológicas y la disponibilidad de los recursos hospitalarios, útil para la toma de decisiones y el establecimiento del mejor tratamiento para una paciente con confirmación o alta sospecha de neoplasia ginecológica.

Palabras clave: cáncer ginecológico, COVID-19, pandemia, complicaciones oncológicas, cáncer.

(C) 2020 Fundación Universitaria de Ciencias de la Salud - FUCS. Este es un artículo Open Access bajo la licencia CC BY-NC-ND (http://creativecommons.org/licenses/by-nc-nd/4.0/).

\section{INFORMACIÓN DEL ARTÍCULO}

Historia del artículo:

Fecha recibido: abril 21 de 2020

Fecha aceptado: abril 27 de 2020
Autor para correspondencia.

Alexander Rodríguez

jorgear12@gmail.com
DOI

10.31260/RepertMedCir.01217372.1047 
The world is experiencing a coronavirus pandemic called SARS-COV-2 which causes coronavirus disease 2019 (COVID 19). This has led to a collapse in health systems, making the management of other diseases a challenge. Similarly, the presence of this disease generates many doubts for oncological groups regarding the provision of standard treatments, which should be carried out as soon as possible, in order to ensure better oncological outcomes.

We propose the creation of an index (COVID 19 Cancer Index) taking into account clinical and epidemiological variables and the availability of hospital resources, which are useful for decision making and determining the best treatment for a patient with confirmed or strongly suspected gynecological neoplasia.

Key words: gynecological cancer, COVID-19, pandemic, oncological complications, cancer.

(C) 2020 Fundación Universitaria de Ciencias de la Salud - FUCS, This is an open access article under the CC BY-NC-ND license (http://creativecommons.org/licenses/by-nc-nd/4.0/).

\section{INT RODUCCIÓN}

La pandemia actual causada por el SARS-COV-2, hasta el 20 de abril de 2020, ha cobrado mas de 2,3 millones de infectados y mas de 170 mil muertes. Hasta ahora las medidas de defensa mas efectivas, incluyen el lavado frecuente de manos, el confinamiento en casa de la población general (lock down), la realización de pruebas de PCR a la población sintomática, pruebas rápidas en suero de $\operatorname{IgG}$ e $\operatorname{IgM}$, asi como el manejo medico experimental y hospitalario para los casos graves. Sin que se conozca hasta el momento un tratamiento curativo en la población vulnerable puede avanzar hasta presentarse como neumonía severa y mortal. La atención de los pacientes contagiados ha requerido una importante ocupación de los recursos humanos, físicos y materiales de los sistemas de salud; incluyendo camas y respiradores artificiales en unidades de cuidados intensivos. Por su parte, la patología por cáncer ha representado a nivel mundial y durante los últimos 10 años la segunda causa de mortalidad, después de la patología cardiovascular. La incidencia, sobrevida y mortalidad varían con el tipo de cáncer, su presentación por estadios y el desempeño del sistema de salud; de tal manera que sólo por cáncer de cuello uterino las cifras llegan a 570 mil casos anuales y aproximadamente 311 mil muertes en 2018. ${ }^{1,2}$

Por ello y por considerarse un problema de salud pública, las sociedades científicas y los pronunciamientos gubernamentales, hacen hincapié en que los pacientes con patología oncológica deben continuar con su tratamiento durante la pandemia. Para el caso específico de las pacientes con sospecha o confirmación de neoplasia ginecológica infiltrante, el tratamiento debe iniciarse lo antes posible, a fin de brindar una mayor posibilidad de sobrevida global y disminuir la tasa de recaídas. Entre los tumores ginecológicos en estadio temprano en que pueden lograrse tasas de sobrevida superiores a $85 \%$, se encuentran el cáncer de cuello uterino, de endometrio y cáncer de vulva, en tanto que el cáncer de ovario cuando se logran citorreducciones R0, la sobrevida a 5 años puede sobrepasar $50 \%$ de acuerdo con el estadio en el que se aborda. ${ }^{3,4,5}$ No obstante, se han sugerido rutas alternas para el manejo de estas pacientes, tales como postergar las cirugías oncológicas tanto como sea posible o cambiar el tratamiento a radioterapia $\mathrm{y} / \mathrm{o}$ quimioterapia si es viable. Basándose en las complejas situaciones que atraviesan las ciudades más afectadas Ramírez y col. brindan varias recomendaciones y escenarios clínicos en enfermedad invasiva para la toma de decisiones en cáncer de cérvix, endometrio y ovario. ${ }^{6}$

Pese a esto no se cuenta con una escala que en el contexto de la pandemia, permita la toma objetiva de decisiones en los planes de tratamiento y su transferibilidad. El presente artículo pretende ofrecer una herramienta de apoyo a la clínica en la toma de decisiones y el establecimiento del mejor tratamiento para una paciente con confirmación o alta sospecha de neoplasia ginecológica.

PROPUESTA DE UN ÍNDICE PARA

MANEJO QUIRÚRGICO DE PACIENTES

CON CÁNCER GINECOLÓGICO EN LA PANDEMIA POR COVID-19

El Índice que se propone surge durante y para la pandemia de COVID-19, motivado por las experiencias diarias en atención a los diferentes casos de neoplasia ginecológica y en diferentes escenarios de la pandemia. Es importante aclarar que debido a la rápida expansión y la consecuente premura, este Índice no ha pasado por el proceso de validación. El presente instrumento, tiene como objetivo brindar una herramienta de cálculo que apoye al equipo oncológico en la elección del mejor tratamiento para 
cada paciente con cáncer ginecológico, donde además de los protocolos de manejo aceptados según la condición clínica, se cuantifiquen otros factores determinantes como la fase epidémica en el contexto de la paciente y los recursos con que cuenta el sistema de salud para hacer frente a dicha epidemia. $^{7}$

Este instrumento permite situar objetivamente el escenario en que se encuentra la paciente y definir la opción de manejo más apropiada. Las variables a tener en cuenta son: el factor epidemiológico, es decir la fase en que se encuentra la epidemia; la infraestructura con que se cuenta, esto es unidad de cuidados intensivos y equipos de protección personal; el recurso humano efectivo y los factores clínicos: edad, estadio de la enfermedad oncológica, y patologías crónicas no controladas. A cada variable se le asigna una calificación que va entre cero y dos puntos y la sumatoria corresponde al COVID-19 Cáncer Index, cuyo valor o intervalo proponen una ruta de tratamiento. ${ }^{8}$

Los factores clínicos como la edad y enfermedades crónicas asociadas como cardiovasculares, diabetes, enfermedades pulmonares crónicas, hipertensión arterial, patologías renales o estados de inmunosupresión, aumentan la probabilidad de complicaciones y de muerte por COVID-19; por lo tanto se deben tener en cuenta a la hora de definir el tratamiento de una paciente oncológica, pues en caso de tener la enfermedad al momento de la cirugía siendo asintomático para COVID-19 o si la adquiere en el posoperatorio puede presentar complicaciones mayores. ${ }^{9}$

\section{FACTOR EPIDEMIOLÓGICO}

Para este índice se considera factor epidemiológico la fase de la epidemia COVID-19 en que se encuentra la región específica o ciudad donde es atendida la paciente.

Fase I preparación: momento en el cual se sabe que el virus va a llegar a la región o se han confirmado pocos casos, todos ellos importados. Se están tomando las medidas para ampliar la capacidad instalada en los servicios de salud; se realiza vigilancia y prevención del contagio y no se emplea una proporción significativa de los recursos en salud en la atención de los casos de COVID-19.

Fase II mitigación: corresponde a la etapa en que se confirman casos importados y casos de transmisión por contacto o nexo epidemiológico. En esta fase predomina la utilización de la capacidad instalada en los pacientes con COVID- 19. Los servicios de salud pueden verse saturados de acuerdo con la eficiencia de la fase de preparación y la velocidad de diseminación de la infección.

Fase III contagio comunitario: diseminación de la enfermedad por transmisión local; esto es que más de $10 \%$ de los casos confirmados carecen de un nexo epidemiológico.
Hay saturación de los servicios de salud con pacientes en estados críticos de esta infección; de forma tal que se limita el uso de los mismos para pacientes con otras patologías, como el cáncer.

Puntaje asignado a cada fase de la epidemia:

- Fase I, Preparación: 0 puntos

- Fase II, Mitigación: 1 punto

- Fase III, Contagio comunitario: 2 puntos

\section{INFRAESTRUCT URA}

Hace referencia a la capacidad de reacción de la región ante la epidemia. Está comprendida por: la capacidad instalada en unidades de cuidado intensivo UCI; la disponibilidad de equipos de protección personal y la suficiencia del recurso humano en salud.

Ocupación de camas de UCI:

- Menor de 30\%: 0 puntos

- Entre el 30 y 50\%: 1 punto

- Mayor a 50\%: 2 puntos

DISPONIBILIDAD DE EQUIPO DE PROTECCIÓN PERSONAL PPE

- Buena disponibilidad: 1 punto

- Poca disponibilidad: 2 puntos

\section{RECURSO HUMANO}

Debido a la movilización del personal hacia la atención de pacientes con COVID-19, se evalúa la suficiencia del talento humano en salud en el momento específico de la toma de decisiones.

- La institución donde se encuentra tiene suficiente personal para la atención de pacientes con COVID-19 y pacientes con cáncer: 1 punto

- La institución tiene escases de personal para atención de COVID-19 y cáncer: 2 puntos

\section{FACTORES CLÍNICOS}

Las variables a considerar son: edad, estadio de la enfermedad oncológica y patologías crónicas no controladas

Edad de la paciente:

- Menor de 50 años: 0 puntos

- Entre 50 y 70 años: 1 punto

- Mayor de 70 años: 2 puntos

Estadio de la enfermedad:

- Estadio temprano: 0 puntos

- Estadio avanzado: 2 puntos 


\section{PATOLOGÍAS CRÓNICAS NO}

CONTROLADAS

Se consideran para este índice: hipertensión arterial no controlada, diabetes descompensada, otros estados de inmunosupresión (tabla 1).

- Ausente: 0 puntos

- Controlada: 1 punto

- No controlada: 2 puntos

Tabla 1. COVID-19 Cáncer Index, para definir manejo quirúrgico en pacientes con tumores ginecológicos.

\begin{tabular}{|l|c|c|c|}
\hline \multicolumn{1}{|c|}{ Criterio } & \multicolumn{3}{|c|}{ Puntaje } \\
\cline { 2 - 4 } Fase epidemiológica & Fase I & Fase II & Fase III \\
Ocupación de UCI & $<30 \%$ & $30-50 \%$ & $>50 \%$ \\
Disponibilidad PPE & N/A & Adecuado & Inadecuado \\
Recurso humano médico & N/A & Disponible & $\begin{array}{c}\text { Asignado a } \\
\text { pacientes con } \\
\text { COVID-19 }\end{array}$ \\
Edad & $<50$ & $50-70$ & $>70$ \\
Estadio tumoral & Temprano & N/A & Avanzado \\
Enfermedades crónicas & Ausente & Controlada & No controlada \\
\hline
\end{tabular}

N/A: No aplica

PPE: Equipo de protección personal

\section{INTERPRETACIÓN DEL COVID-19} CÁNCER INDEX

Menor de 5: proceder a manejo estándar incluido manejo quirúrgico.

Entre 6 y 9: considerar postergar manejo quirúrgico por seis semanas o iniciar manejo con quimioterapia $\mathrm{y} / \mathrm{o}$ radioterapia, según la patología.

Entre 10 y 14: postergar manejo quirúrgico por seis semanas o iniciar manejo con quimioterapia y/o radioterapia, según la patología. Se sugiere remisión a centro oncológico con mayor disponibilidad de recursos.

\section{Casos clínicos ejemplo 1:}

Paciente de 54 años asintomática, con diagnóstico de cáncer de cuello uterino invasivo, estadio IBl, tumor de $15 \mathrm{~mm}$, resonancia de abdomen y pelvis negativa para enfermedad metastásica, sin patologías crónicas. Situación epidémica de la ciudad en fase I. El centro médico donde se encuentra la paciente cuenta con buenos recursos de PPE y humanos, la ocupación de camas de cuidados intensivos es de $20 \%$.

\section{Resultado del COVID-19 Cáncer Index: 3}

Con este resultado la paciente se debe llevar a tratamiento estándar el cual es histerectomía radical con linfadenectomía pélvica o disección de ganglio centinela pélvico lo antes posible, con intención curativa.

\section{Casos clínicos ejemplo 2:}

Paciente de 68 años, con tomografía de abdomen total que reporta tumor pélvico de $15 \mathrm{~cm}$ e implantes en omento y peritoneo (por imágenes avanzado), sin ascitis, CA 125 de 250 $\mathrm{U} / \mathrm{ml}$, hipertensa controlada. Ciudad en fase epidémica II, con ocupación de UCI de 40\%, personal humano disponible, y los equipos de PPE comienzan a ser insuficientes.

Resultado del COVID-19 Cáncer Index: 9

Con este resultado, y ante la alta sospecha de un carcinoma de ovario estadio III, se sugiere no llevar a cirugía inicialmente. La recomendación es realizar biopsia percutánea, confirmar malignidad e iniciar tratamiento con quimioterapia neoadyuvante para posteriormente y según la respuesta al tratamiento, definir cirugía de intervalo.

Cada caso es diferente y se ubica en un puntaje de índice igualmente diferente, que permite al equipo tratante definir el manejo más adecuado según las variables analizadas.

\section{CONCLUSIONES}

En el momento actual de pandemia por COVID-19, tomar conductas para el tratamiento de pacientes con neoplasias ginecológicas puede ser difícil, por lo cual debemos evaluar los diferentes factores que ubican a la situación de la paciente, en un momento determinado, con el fin de no alterar el pronóstico de la enfermedad, y así poder brindar el mejor tratamiento oncológico a cada caso.

El COVID-19 Cáncer Index, puede resultar útil para la toma de decisiones en pacientes con cáncer o sospecha de cáncer ginecológico en el contexto de la pandemia actual por coronavirus; además también podría ser útil para otros tumores, realizando las adaptaciones según la patología.

\section{REFERENCIAS}

Bray F, Ferlay J, Soerjomataram I, Siegel RL, Torre LA, Jemal A. Global cancer statistics 2018: GLOBOCAN estimates of incidence and mortality worldwide for 36 cancers in 185 countries. CA: a cancer journal for clinicians. 2018;68(6):394-424. doi: 10.3322/ caac. 21492 
2. Arbyn M, Weiderpass E, Bruni L, de Sanjosé S, Saraiya M, Ferlay J, et al. Estimates of incidence and mortality of cervical cancer in 2018: a worldwide analysis. The Lancet Global Health. 2020;8(2):e191-e203. doi: http://doi.org/10.1016/s2214$109 x(19) 30482-6$

3. Ramirez PT, Frumovitz M, Pareja R, Lopez A, Vieira M, Ribeiro R, et al. Minimally Invasive versus Abdominal Radical Hysterectomy for Cervical Cancer. The New England journal of medicine. 2018;379(20):1895-904. doi: http://doi.org/10.1056/ NEJMoa 1806395

4. Walker JL, Piedmonte MR, Spirtos NM, Eisenkop SM, Schlaerth JB, Mannel RS, et al. Recurrence and survival after random assignment to laparoscopy versus laparotomy for comprehensive surgical staging of uterine cancer: Gynecologic Oncology Group LAP2 Study. Journal of clinical oncology: official journal of the American Society of Clinical Oncology. 2012;30(7):695-700. doi: http://doi.og/10.1200/JCO.2011.38.8645.

5. Fader AN, Rose PG. Role of surgery in ovarian carcinoma. Journal of clinical oncology : official journal of the American Society of Clinical Oncology. 2007;25(20):2873-83. doi: http://doi. og/10.1200/JCO.2007.11.0932
6. Ramirez PT, Chiva L, Eriksson AGZ, Frumovitz M, Fagotti A, Gonzalez Martin A, et al. COVID-19 Global Pandemic: Options for Management of Gynecologic Cancers. International journal of gynecological cancer: official journal of the International Gynecological Cancer Society. 2020. doi: http://dx.doi. org/10.1136/ijgc-2020-001419

7. World Health Organization. Closing the gap in a generation: Health equity through action on the social determinants of health. Geneva: World Health Organization; 2008. p. 40.

8. Pan American Health Organization-PAHO. COVID 19 Recommendations for medical surge capacity and deployment of e mergency m edical t eams. Pan American Health Organization; 2020. p. 9.

9. Wang L, He W, Yu X, Hu D, Bao M, Liu H, et al. Coronavirus disease 2019 in elderly patients: Characteristics and prognostic factors based on 4-week follow-up. The Journal of infection. 2020. doi: https://doi.org/10.1016/j.jinf.2020.03.019 